\title{
Investigadores y cultura estadística: Una mirada crítica
}

\author{
Researchers and Statistical culture: A critical sight
}

Pesquisadores e cultura estatística: um olhar crítico

\author{
Elizabeth Gandica-de Roa ${ }^{\mathrm{a}^{*}}$ \\ ${ }^{a}$ Doctora en Educación-Universidad Nacional Experimental del Táchira. Unet. San Cristóbal, Venezuela. \\ Orcid: 0000-0001-8797-4198
}

Forma de citar: Gandica, E. (2017). Investigadores y cultura estadística: Una mirada crítica. Eco matemático 8(1). 15-24

Recibido: abril 2 de 2016

Aceptado: julio 10 de 2016

\begin{abstract}
Palabras clave
Comunidad de Investigadores, Cultura estadística y Razonamiento estadístico.
\end{abstract}

\section{Keywords}

Community of Researchers, Statistical Culture and Statistical Reasoning.

\begin{abstract}
Resumen: El término "Cultura Estadística" está referido a la capacidad y conocimiento que debe tener un individuo o grupo social, para entender, comprender y analizar el dato y la información estadística. Estudiar la cultura estadística en un grupo de individuos supone medir el conocimiento y la forma como se llevan a cabo las aplicaciones estadísticas, determinar el nivel de razonamiento y describir la actitud hacia la misma. En la presente investigación se analizaron estos componentes en la comunidad de investigadores de dos universidades venezolanas. La comunidad de investigadores quedo conformada por tutores, jurados y/o asesores de trabajos de grado que laboran en el decanato de Postgrado de cada universidad. La investigación se llevó a cabo bajo el enfoque cuantitativo. La recolección de datos se realizó a través de la técnica de la encuesta y la prueba. Los instrumentos de recolección de datos fueron: el test de la taxonomía "SOLO", el test de actitudes "ATS" y la prueba objetiva. El análisis de datos se realizó con: análisis descriptivo y análisis factorial de componentes principales, encontrándose que el razonamiento estadístico en los investigadores se encuentra en los niveles uniestructural y preestructural; el conocimiento en un nivel básico; las aplicaciones estadísticas realizadas son incorrectas y la actitud es favorable hacia la estadística, con énfasis en una buena disposición hacia el aprendizaje y actualización de los conocimientos.
\end{abstract}

Analizar la Cultura Estadística, evidenció los aspectos de la Estadística, que acompañan el desarrollo de las diversas investigaciones académicas producidas en el entorno. Este conocimiento delimitará y fortalecerá los cimientos metodológicos estadísticos, que afronta el desarrollo de toda investigación científica.

\begin{abstract}
The term "Statistical Culture" refers to the capacity and knowledge that an individual or social group must have to understand, understand and analyze the data and statistical information. Studying the statistical culture in a group of individuals involves measuring knowledge and the way in which statistical applications are carried out, determining the level of reasoning and describing the attitude towards it. In the present investigation, these components were analyzed in the community of researchers from two Venezuelan universities. The community of researchers is made up of tutors, juries
\end{abstract}

\footnotetext{
* Autor para correspondencia egandica@unet.edu.ve

http://dx.doi.org/10.22463/17948231.1471
} 
and / or advisors of degree works who work in the Postgraduate Deanship of each university. The research was carried out under the quantitative approach. The data collection was done through the technique of the survey and the test. The data collection instruments were: the "SOLO" taxonomy test, the "ATS" attitude test and the objective test. The data analysis was carried out with: descriptive analysis and factorial analysis of the main components, finding that the statistical reasoning in the researchers is at the uniestructural and prestructural levels; knowledge at a basic level; the statistical applications made are incorrect and the attitude is favorable towards statistics, with emphasis on a good disposition towards learning and updating knowledge.

Analyze the Statistical Culture, evidenced the aspects of Statistics, that accompany the development of the diverse academic researches produced in the environment. This knowledge will delimit and strengthen the statistical methodological foundations, which face the development of all scientific research.

\section{Palavras-chave}

Comunidade de Pesquisadores, Cultura Estatística e Raciocínio Estatístico
Resumo: O termo "Cultura Estatística" refere-se à capacidade e conhecimento que um indivíduo ou grupo social deve ter, para compreender, compreender e analisar dados e informações estatísticas. Estudar a cultura estatística em um grupo de indivíduos envolve medir o conhecimento e a maneira pela qual as aplicações estatísticas são realizadas, determinando o nível de raciocínio e descrevendo a atitude em relação a ele. Na presente investigação, esses componentes foram analisados na comunidade de pesquisa de duas universidades na Venezuela. A comunidade de pesquisa foi composta por tutores, jurados e / ou avaliadores de trabalhos de graduação que atuam no decanato de pós-graduação de cada universidade. A pesquisa foi realizada sob a abordagem quantitativa. A coleta de dados foi realizada através da técnica de pesquisa e teste. Os instrumentos de coleta de dados foram o teste de taxonomia "SOLO", o teste de atitude "ATS" e o teste objetivo. A análise dos dados foi realizada com: análise descritiva e análise fatorial dos componentes principais, constatando que o raciocínio estatístico dos pesquisadores está nos níveis não estrutural e preestructural, conhecimento em nível básico, as aplicações estatísticas realizadas são incorretas e a atitude é favorável à estatística, com ênfase na disposição de aprender e atualizar o conhecimento.

Analisando a Cultura Estatística, evidenciaram-se os aspectos da Estatística, que acompanham o desenvolvimento de diversas pesquisas acadêmicas produzidas no ambiente. Esse conhecimento irá delimitar e fortalecer a fundamentação estatística metodológica, que aborda o desenvolvimento de toda a pesquisa científica.

\section{Introducción}

El papel de las Universidades ha venido cambiando con el transcurrir de los años. Estas se han visto en la imperiosa necesidad de abordar la educación no solo en el ámbito de enseñar contenidos, sino de preparar a los nuevos profesionales en el área de la investigación, esto con el fin de dotarlos de herramientas procedimentales, conceptuales y actitudinales, que les permita la producción científica en aras de mejorar y satisfacer las necesidades del entorno en que se encuentran inmersos. La calidad de la educación universitaria según Bracho (2011), está asociada a las prácticas investigativas, las cuales deben generar una cultura investigativa y una producción científica en las comunidades de investigadores de la Universidad. Son estas, las que deben hacer investigación, consumir investigación y utilizar esta investigación, en pro del beneficio de la sociedad y de la comunidad universitaria.

Para Leal (2009), la comunidad de investigadores está constituida; por docentes, tutores, asesores e investigadores, quienes deben tener experiencia en investigación, para así lograr en los tutorados, 
asesorados o participantes su desarrollo científico. Leal, manifiesta que en la actualidad, existe una deficiencia en las prácticas investigativas, que interfiere de forma negativa en la producción científica por parte de la comunidad de investigadores de las Universidades. Esta problemática es visualizada por Bracho, cuando manifiesta que en el Estado Zulia: "según observaciones no sistémicas de docentes y participantes, existe deficiencias en la aplicación de las herramientas metodológicas para acercarse a la realidad objeto de estudio".

En este orden de ideas, la investigadora realizó una revisión de la praxis investigativa en dos universidades venezolanas ubicadas en el estado Táchira El análisis se llevó a cabo en algunos de los productos científicos producidos en ambas universidades, se revisaron 70 trabajos de grado presentados en las distintas maestrías que ofrecen estas universidades, realizados estos bajo el enfoque cuantitativo; y 15 artículos científicos que forman parte de su base de datos personal originada en trabajos como jurado evaluador $\mathrm{y}$ árbitro. El análisis se realizó en tres dimensiones: a) Técnico - científica, referido este a los elementos de contenido, métodos estadísticos aplicados, selección de la población y muestra, análisis de datos, entre otros, b) forma, la cual comprende los aspectos relacionados con el uso de lenguaje y de presentación; y c) implicaciones, que aluden al posible impacto potencial de la investigación a corto y mediano plazo.

En cada dimensión se incluyeron ciertos criterios para la evaluación. Los resultados de este análisis evidenciaron que en la dimensión Técnicocientífica, dimensión que servirá de referente a la problemática de esta investigación, el 90\% de los trabajos de grado y artículos científicos presentan debilidades.

Tales debilidades se refieren a: supuestos teóricos no comprobados para los modelos estadísticos aplicados, abusos en el uso del teorema del Limite Central, técnicas de muestreo mal seleccionadas, uso de fórmulas de muestreo no acordes a la investigación, errores en el marco muestral, errores en la definición de la población de estudio, ausencia de pruebas de bondad de ajuste, errores en la interpretación de las medidas calculadas, ausencia de las teorías de estimación cuando son necesarias, entre otros.

Es de importancia resaltar, que en los trabajos de grado y artículos revisados se pudo evidenciar que los investigadores apoyan sus trabajos, como así consta en la bibliografía citada por ellos, en los libros de metodología de la investigación cuantitativa, en investigaciones previas y en los conocimientos que poseen sus tutores o asesores. Se procedió a realizar una revisión de algunos libros de metodología de la investigación cuantitativa, encontrando que en estos, están presentes también las debilidades antes mencionadas, es decir, debilidades en el procedimiento estadístico, teorías de muestreo ausentes o incompletas, ausencia de fórmulas de muestreo, poca rigurosidad matemática en el planteamiento de los métodos estadísticos y ausencia de la teoría de estadística inferencial, pues se hace más énfasis en las aplicaciones de la estadística descriptiva, como se evidencia, por ejemplo, en el libro de Metodología de la Investigación Cuantitativa, del autor Santa Palella Stracuzzi (2004), en el capítulo de Tratamiento y análisis de la información, página 163.

Ahora bien, a pesar de la problemática presentada, los productos científicos antes citados, se encuentran en las bibliotecas o están publicados; y sirven como referenciales e incluso como fuentes secundarias, para otras investigaciones, logrando así, que estos errores se transfieran de investigación a investigación, por lo cual, es de esperar que algunas conclusiones o toma de decisiones adolezcan de fiabilidad. Por otro lado, es importante destacar que, de no estudiarse esta situación y proponerse alternativas de solución, en el menor tiempo posible, se seguirán afectando las investigaciones futuras, al punto que, sus resultados no merezcan la confiabilidad que exige 
su potencial servicio a la utilización de referentes o fuentes secundarias.

Cabe entonces preguntarse: ¿Serán los investigadores los que no consultan libros de estadística, o será que no los citan en sus trabajos? ¿Será que el paradigma dominante de la ciencia tiene que ver en algo sobre la forma en que se colocan los contenidos en estos libros, o será el anacronismo de los autores, de los investigadores, de los tutores, de los manuales o de los estilos de cada universidad? ¿Existe falta de conocimiento de la estadística? ¿Cuál es el nivel de conocimiento de los investigadores? ¿Cuál es la actitud de estos frente a la estadística? ¿Cómo fue la experiencia como estudiantes? ¿Cómo han desarrollado el pensamiento estadístico?

Velázquez (2008), señala que los investigadores tienen dificultades a la hora de concebir y socializar con los resultados obtenidos en sus investigaciones, debido a que no existe bibliografía al respecto, y por lo regular, en los manuales de Metodología de la Investigación, se dedica la atención a los elementos de diseño técnico metodológico, al proceso, sus etapas y la elaboración del informe de la investigación, pero no se hace referencia a cómo elaborar la propuesta y qué alternativas pueden presentar los aportes como resultados científicos. Esto explica que al revisar los productos científicos, como trabajos de grado de maestría, doctorado, artículos, entre otros, se aprecie una confusión en el uso de la terminología al proponer la solución o resultado principal de la investigación.

Para Batanero (2001), la problemática encontrada en el aspecto técnico-metodológico en las investigaciones, tiene su origen en el escaso desarrollo de la cultura estadística, la cual no ha logrado dar a conocer a profesionales, estudiantes, usuarios públicos y al público en general qué es o qué hace la estadística.

En la búsqueda de las posibles explicaciones a la falta de conocimiento en estadística, es necesario identificar las tres perspectivas de abordaje de esta problemática: ¿Que saben de estadística los investigadores?, ¿Cuál es el nivel de razonamiento estadístico? y ¿cuál es la actitud hacia esta disciplina?

Para dar solución a lo planteado, la investigadora se propuso analizar los componentes de la cultura estadística, en la comunidad de investigadores de dos universidades venezolanas. Este abordaje permitirá, trascender la escisión que se plantea en algunos estudios entre el contenido disciplinario y la relación investigativa.

Para llevar a cabo esta investigación se consideraron algunos estudios previos relacionados al conocimiento, razonamiento y actitud hacia la estadística como disciplina. Pinto (2010), estudió el conocimiento didáctico del contenido sobre la representación de datos estadísticos, tuvo como objetivos describir las concepciones que tienen los profesores sobre la Estadística, su enseñanza y aprendizaje, así como el conocimiento que tienen del tópico, de las estrategias de aprendizaje y del conocimiento del estudiante sobre la representación gráfica en Estadística. Utilizó una prueba objetivo con dimensiones e indicadores y encontró que el conocimiento de cada profesor está influenciado por su concepción hacia la estadística y la matemática. La metodología utilizada por Pinto, es aplicable a esta investigación para clasificar el conocimiento en la comunidad de investigadores, el cual es uno de los componentes de la cultura estadística.

Para analizar el razonamiento estadístico se utilizó el modelo de taxonomía SOLO, aplicado por Duarte (2014), en su estudio de comprensión y razonamiento de profesores de Matemáticas de bachillerato sobre conceptos estadístico básico, cuyo resultado evidenció que los profesores evaluados tienen una comprensión superficial y aislada sobre conceptos estadísticos y el razonamiento estadístico se ubica en los niveles más bajos del modelo.

En cuanto a la medición de las actitudes se utilizó como metodología la aplicada por Estrada (2012), el cual analizó las actitudes de los profesores y su influencia en los estudiantes con el Test de la escala 
de actitudes ATS; Attitudes Toward Statistics, creada por Wise (1985), que mide dos dimensiones de actitudes: una actitud hacia la estadística y otra hacia el curso. Este estudio pretendió medir no solo la vinculación de las actitudes con el logro, sino los factores que constituyen las actitudes hacia la Estadística. Una vez analizada la influencia de todas estas variables, concluye que las actitudes hacia la estadística tienden a ser negativas y que la variable que tiene mayor peso en todos los factores que constituyen las actitudes hacia la estadística, así como las actitudes generales ante esta materia, es la motivación.

Dado que el eje central de esta investigación es la cultura estadística, se explicará brevemente algunos parámetros que servirán de eje conceptual para entender la interpretación y explicación de la investigación

La estadística puede ser considerada una disciplina, cuyos métodos y aplicaciones han permeado la mayoría de las áreas de la ciencia. La realidad es que se ha convertido en una disciplina que evolucionó, para quedarse e incorporarse a la cultura de la sociedad moderna. Actualmente, la estadística está mucho más relacionada con otras disciplinas que las matemáticas. Se ha usado como lenguaje y método de investigación científica, en áreas tan diferentes como la lingüística, geografía, física, ingeniería, psicología y economía. El conjunto de habilidades estadísticas básicas que una persona necesita para manejar información en el día a día se refiere como «cultura estadística».

El modelo de Gal (2002) permite estudiar la cultura estadística desde dos perspectivas: elementos del conocimiento que involucra conocimiento estadístico y habilidades para lograr razonamientos lógicos - deductivos, y elementos disposicionales que tienen que ver con las creencias, actitudes y las posturas críticas.

Los elementos del conocimiento son aquellos que permiten verificar si un individuo tiene dominio de la disciplina estadística. Estos conocimientos tienen que ver con la utilidad de los datos, la forma en que se producen, los términos e ideas básicos relacionados con estadística descriptiva, ideas relacionadas a las gráficas y tablas, comprensión de las medidas de tendencia central, posición, variabilidad y forma, y conocimiento de cómo se llega a conclusiones e inferencias estadísticas.

En cuanto al razonamiento estadístico, este es definido según Garfield, Del Mas y Chance (2003), como la manera que las personas razonan con ideas estadísticas y el sentido que le dan a la información estadística. Esto implica hacer interpretaciones, conectar un concepto con otro, entender y poder explicar los procesos estadísticos.

Referente a los elementos disposicionales en los que se encuentran las actitudes, Rockeach (1968), las define como «una organización de creencias relativamente permanentes que predisponen a responder de un modo preferencial ante un objeto o situación»».

\section{Materiales y métodos}

La investigación se desarrolló bajo un enfoque cuantitativo, no experimental, descriptivo de campo. La población quedó conformada por los tutores, jurados y/o asesores de los trabajos de grado, que laboran en el decanato de postgrado de dos universidades venezolanas, ubicadas en el estado Táchira. La muestra objeto de estudio quedó constituida por 78 tutores, asesores y/o jurados en las dos universidades, seleccionados aleatoriamente y en forma estratificada con asignación proporcional por universidad y por maestría.

Como técnicas de recolección de datos se utilizó la encuesta y la prueba. Se aplicaron como instrumentos el modelo de taxonomía "SOLO", para analizar el razonamiento estadístico, el test de actitudes "ATS" y la prueba objetiva para estudiar el nivel de conocimiento.

En el modelo SOLO, los conceptos y procesos utilizados por los sujetos al dar respuesta a las preguntas o tareas planteadas se pueden clasificar en un determinado nivel de los cinco niveles que se contempla.) Pre-estructural: las respuestas que 
aporta el evaluado ante una determinada tarea son erróneas o inexistentes. Los participantes no tienen entendimiento. Utilizan contestaciones evasivas o están totalmente equivocados. b) Uni-estructural: la respuesta dada por el evaluado sólo se centra en un determinado aspecto que, por otro lado, no tiene por qué ser relevante. Los participantes solo cumplen una parte de la tarea, se quedan en la terminología. c) Multi-estructural: el evaluado es capaz de enumerar una serie de aspectos correctos, pero no va más allá. Los participantes dan un conjunto detallado de datos pero no los relaciona. d) Relacional: el evaluado no sólo se centra en varios aspectos correctos sino que también es capaz de relacionarlos entre sí. Los participantes son capaces de dar una explicación de la relación. Hay comprensión, y e) Abstracto ampliado: es el nivel más complejo, el evaluado cumple con los anteriores criterios y, además, es capaz de ir más allá de lo preguntado para poder relacionarlo con otros sistemas ajenos a la tarea en sí pero que, de algún modo, enriquecen la respuesta.

El instrumento para aplicar el modelo SOLO quedó formado por nueve ítems, los cuales miden cada nivel del modelo. Tabla 1. La clasificación se obtuvo a través de un análisis descriptivo en términos porcentuales.

Tabla 1. Clasificación de los items del cuestionario para los niveles de razonamiento de modelo de taxonomía "solo"

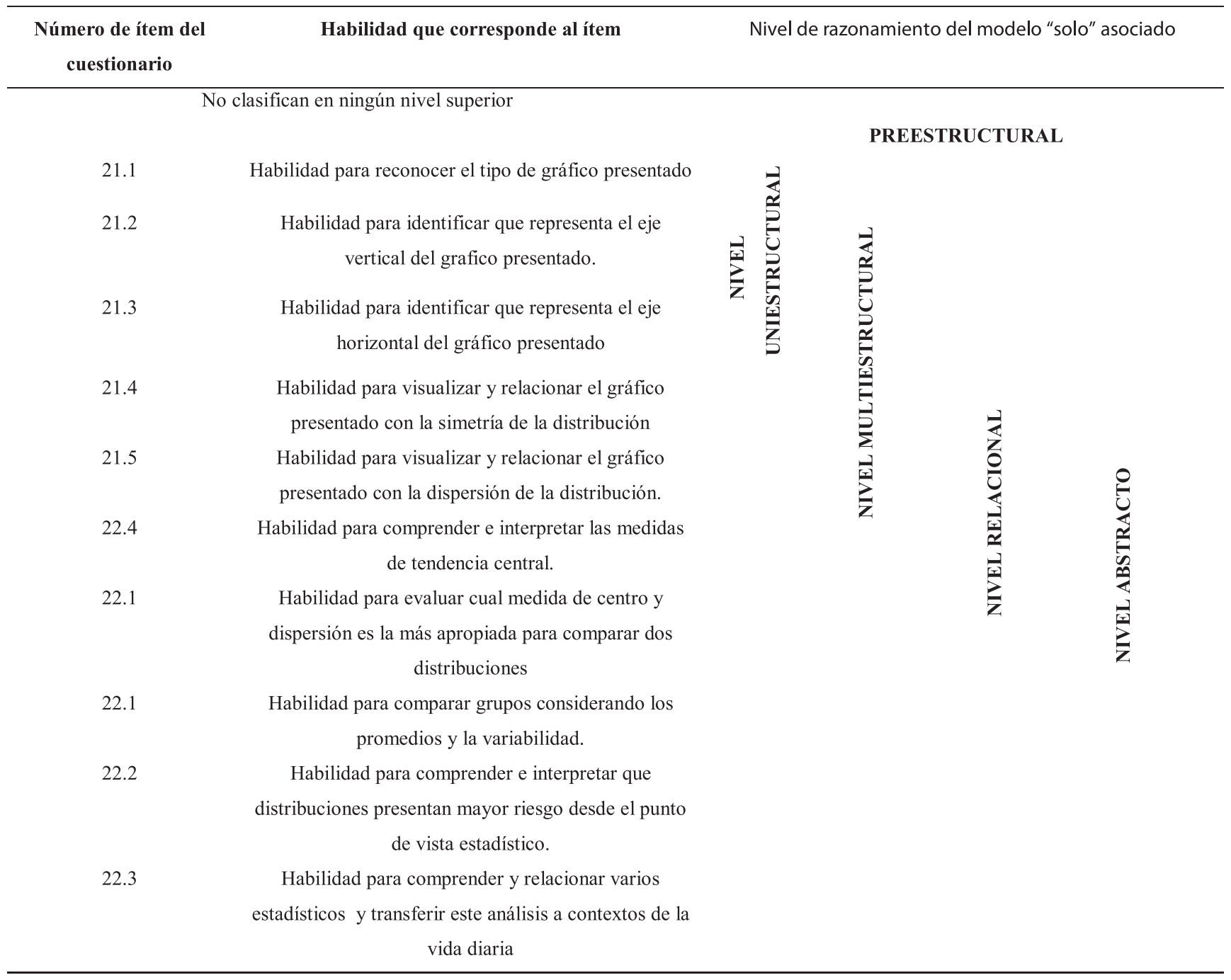

Fuente: Autor 
Para medir las actitudes se aplicó el modelo de medición de actitud hacia la estadística (ATS), propuesto por Wise en 1985. El instrumento consistió de 17 afirmaciones desfavorables y 11 afirmaciones favorables. Para la escala de medición se utilizó escala Likert de 5 categorías. La opción 1 representa el muy de acuerdo, la opción 2 de acuerdo, la opción 3 medianamente de acuerdo, la opción 4 poco de acuerdo y la opción 5 nada de acuerdo. Se redactaron 9 ítems para el componente afectivo, 11 ítems para el componente cognitivo y 8 ítems para el componente conductual.

Se utilizó análisis factorial de componentes principales para el test "ATS", método que permitió determinar cuál es la mejor combinación lineal de las variables. Mejor, en el sentido de que una combinación lineal particular de las variables sea capaz de extraer la mayor cantidad de varianza en los datos. De acuerdo con lo anterior, el primer componente puede ser visto como el mejor resumen simple de combinación lineal. El segundo componente es definido, como la segunda mejor combinación lineal de las variables, bajo la condición de que el segundo componente sea ortogonal con el primero. Los componentes subsiguientes son definidos de manera análoga hasta que toda la varianza es extraída de los datos. Con este criterio se toman para el análisis aquellos componentes que expliquen la mayor parte de la variación de los datos.

Con respecto a la prueba objetiva esta se aplicó para analizar el nivel de conocimiento, quedó conformada de 20 ítems de preguntas cerradas, que evidenciaron el conocimiento y la aplicación de los procesos estadísticos involucrados en las investigaciones científicas. Las diez primeras preguntas correspondieron a conceptos básicos de la estadística descriptiva, mientras que las otras 10 preguntas a conceptos de estadística inferencial. El criterio de clasificación se detalla con los resultados.

\section{Resultados y discusión}

A continuación se muestran los resultados para cada componente: Conocimiento, razonamiento y actitud hacia la estadística, componentes que definen a la cultura estadística.

\section{Conocimiento:}

Para el análisis de esta dimensión se tomaron las respuestas correctas de los ítems que permiten medir el conocimiento. Se dividió el análisis en dos categorías, una para estadística descriptiva y la otra para estadística inferencial. El puntaje máximo era de 10 aciertos en cada categoría. Se encontró como resultado importante que ningún investigador contestó las preguntas relacionadas a estadística inferencial. Por lo tanto, el análisis se detalla solo para la categoría estadística descriptiva, cuyo criterio de clasificación se muestra en la tabla 2.

Tabla 2. Clasificación del nivel de conocimiento según puntuación obtenida en la prueba objetiva.

\begin{tabular}{lc}
\hline \multicolumn{2}{c}{ CLASIFICACION DEL } \\
CONOCIMIENTO \\
\hline Nivel alto & $8-10$ \\
Nivel medio & $5--7$ \\
Nivel bajo & $0--4$ \\
\hline \multicolumn{2}{c}{ Fuente: Autor }
\end{tabular}

Como resultado de este componente se evidenció que el $100 \%$ de los investigadores no conocen los conceptos y procesos de la estadística inferencial. El 38\% de los tutores y/o jurados tienen un conocimiento básico medio en conceptos $\mathrm{y}$ aplicaciones de estadística descriptiva, mientras que un $54 \%$ posee un conocimiento básico bajo. Solo un $8 \%$ de los tutores y jurados obtuvieron una calificación de 8 puntos o más, ubicándoles en un nivel básico alto. Ver gráfico 1 .

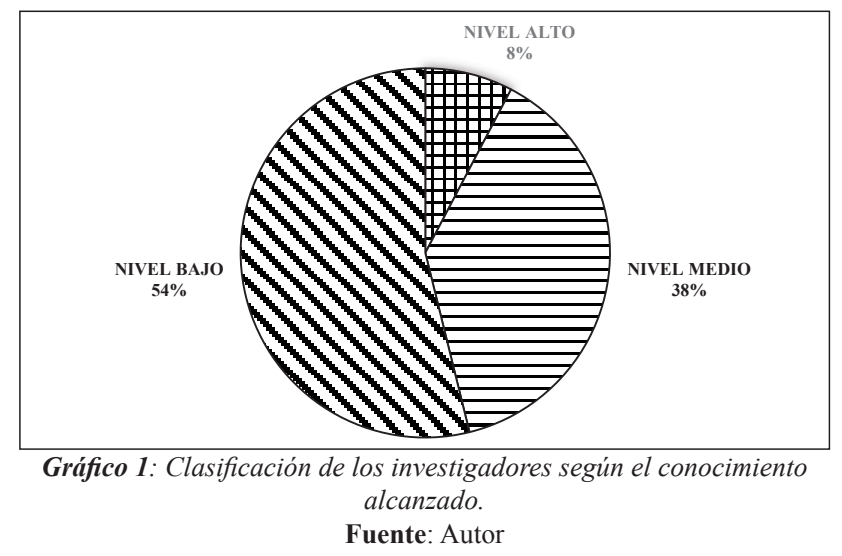




\section{Razonamiento Estadístico}

Para el análisis de esta componente se aplicó el modelo de taxonomía "SOLO", descrito anteriormente. En el gráfico 2, se puede observar que el 39\% quedó ubicado en un nivel pre estructural, el cual corresponde a la no comprensión de los ejercicios planteados, el $52 \%$ de los tutores y/o jurados se encuentran en un nivel de razonamiento uni-estructural, es decir, realizan un procedimiento sencillo para dar respuesta a los planteamientos, el $6 \%$ se ubicó en el nivel multi-estructural, donde los tutores y/o jurados mostraron capacidad de enumerar una serie de aspectos correctos pero no de relacionar; y solo un 5\% quedó en nivel relacional, debido a que no alcanzaron el nivel de comparar y relacionar. Cabe resaltar que ningún profesor está en el nivel abstracto del modelo "SOLO".

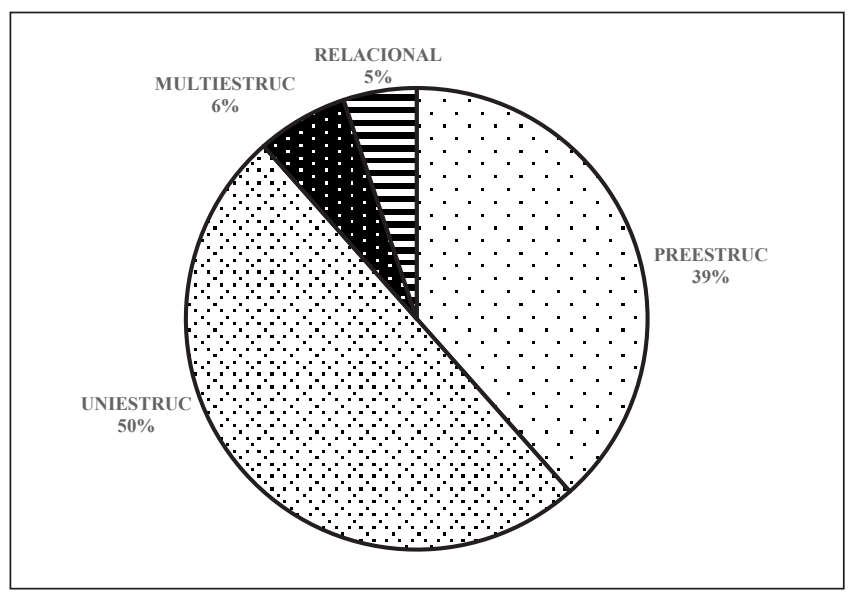

Gráfico 2: Clasificación de los investigadores según el nivel de razonamiento alcanzado.

Fuente: Autor

\section{Actitud}

Para determinar el tipo de actitud en los investigadores se utilizó análisis factorial de componentes principales en los dos grupos de ítems: favorables y desfavorables. Los componentes principales para cada grupo se muestran en las matrices de componente rotado, Tablas 3 y 4.
Tabla 3. Matriz de componente rotado para los items favorables.

\begin{tabular}{ccccc}
\hline \multicolumn{5}{c}{ Componente } \\
& 1 & 2 & 3 & 4 \\
\hline IG &, 864 &, 233 &,- 234 &, 110 \\
IH &, 698 &,- 009 &, 171 &, 410 \\
IO &, 540 &,- 066 &, 274 &,- 154 \\
IX &, 070 &, 841 &,- 015 &, 191 \\
IA &, 415 &, 673 &, 147 &,- 086 \\
IBB &, 384 &,- 614 &, 366 &, 073 \\
IR &, 198 &,- 131 &, 798 &, 079 \\
IM &,- 279 &, 457 &, 647 &, 219 \\
IQ &,- 162 &, 079 &,- 232 &, 710 \\
ID &, 131 &, 184 &, 242 &, 576 \\
IW &, 197 &,- 136 &, 263 &, 560 \\
\hline
\end{tabular}

Fuente: SPSS para Windows. Método de extracción: análisis de componentes principales. Método de rotación: Varimax con normalización Kaiser.

Tabla 4. Matriz de componente rotado para los items desfavorables.

\begin{tabular}{ccccccc}
\hline \multicolumn{7}{c}{ Componente } \\
& 1 & 2 & 3 & 4 & 5 & 6 \\
\hline IAA &, 793 &,- 122 &, 264 & & &, 233 \\
IN &, 678 &, 132 & &, 108 & &,- 351 \\
IZ &, 658 &,- 132 & &,- 204 & &, 228 \\
IT &, 652 &, 168 &,- 461 &, 107 &, 174 & \\
IE & &, 786 &, 251 &,- 143 & & \\
IC & &, 733 & &, 142 &, 460 & \\
IL &,- 198 &, 714 & &, 331 &,- 131 & \\
IV & & &, 776 &,- 171 & &,- 112 \\
IF & &, 403 &, 648 & &, 234 &, 237 \\
IU &, 202 & &, 554 &, 523 &,- 381 &, 126 \\
IS &, 103 &, 400 &, 464 &, 278 & &,- 204 \\
IB & &, 120 & &, 779 &, 122 & \\
IY & & &,- 290 &, 738 &, 223 &,- 228 \\
IJ &,- 215 &, 124 & &, 156 &, 802 &, 181 \\
IK &, 430 & & & &, 693 & \\
II &, 234 &,- 190 & & &, 154 &, 766 \\
IP &, 208 &,- 207 &, 218 &, 365 &, 378 &,- 570 \\
\hline
\end{tabular}

Fuente: SPSS para Windows. Método de extracción: análisis de componentes principales.

Método de rotación: Varimax con normalización Kaiser.

Como resultado se puede concluir que los profesores tienen una actitud general favorable hacia la estadística. Los ítems significativamente representativos son aquellos que tienen puntuaciones superiores a 0,5 en cada componente. Se encontró que 
la mayoría de los tutores y/o jurados tienen una disposición general favorable hacia la estadística, con mayor énfasis en una buena disposición hacia el aprendizaje y actualización de los conocimientos.

Esto se evidencia en las puntuaciones por componente. Tabla 5 y tabla 6 . Para las proposiciones positivas el resultado favorable debe estar cerca de 1 y alejado de 5 , mientras que para las proposiciones negativas lo favorables es cerca de 5 y alejado de 1. Si se observan las puntuaciones ningún componente está en situación crítica, por lo que se concluye que la actitud es favorable.

Tabla 5. Puntuaciones de las componentes principales en los items favorables

DESCRIPCION DE LOS FACTORES QUE COMPONEN LA DIMENSION ACTITUD COMPONENTES AFIRMACIONES POSITIVAS

\begin{tabular}{ccccc} 
& C1 & C2 & C3 & C4 \\
COGINITIVO & 40 & 50 & 50 & 66,6 \\
AFECTIVO & 20 & 50 & 0 & 0 \\
CONDUCTUAL & 40 & 0 & 50 & 33,33 \\
PUNTUACION PROMEDIO & 2,67 & 2,33 & 1,84 & 2,5 \\
\hline & Fuente: Autor
\end{tabular}

Fuente: Autor

Tabla 6. Puntuaciones De Las Componentes Principales En Los Ítems Desfavorables

DESCRIPCION DE LOS FACTORES QUE COMPONEN LA DIMENSION ACTITUD COMPONENTES AFIRMACIONES NEGATIVAS

\begin{tabular}{cccccc} 
& C1 & C2 & C3 & C4 & C5 \\
COGINITIVO & 60 & 33,33 & 50 & 0 & 33,33 \\
AFECTIVO & 20 & 33,33 & 25 & 33,33 & 33,33 \\
CONDUCTUAL & 20 & 33,33 & 25 & 66,66 & 33,33 \\
PUNTUACION PROMEDIO & 4,07 & 4,15 & 3,60 & 4,53 & 4,44 \\
\hline
\end{tabular}

Fuente: Autor

Es importante resaltar como en el componente 3 del grupo de afirmaciones desfavorables, los tutores y/o jurados manifiestan que la estadística es una materia complicada, que requiere de mucho cálculo y que les causa miedo. Asimismo en los componentes 1 y 4 del grupo de afirmaciones favorables, los tutores $\mathrm{y} / \mathrm{o}$ jurados no perciben la estadística como requisito en su práctica académica, consideran que tener habilidades en los procedimientos estadísticos no les facilitará el acceso al desarrollo de investigaciones, las fórmulas estadísticas no les resultan fáciles de entender y no les parece que sea fácil aprender estadísticas.

\section{Conclusiones}

La presente investigación, permitió analizar la cultura estadística en la comunidad de investigadores de dos universidades venezolanas.

En virtud de los resultados obtenidos y en relación con los objetivos específicos planteados, la investigación permite arrojar las siguientes conclusiones:

1. En la componente conocimiento, se concluye que los tutores y/o jurados tienen un conocimiento básico, en estadística descriptiva. No conocen conceptos ni procesos de estadística inferencial. Los tutores y/o jurados entienden la utilidad de la estadística en las investigaciones científicas, la definen en un plano descriptivo, como una herramienta para analizar, ordenar y cuantificar datos. Poseen un conocimiento básico, aunque hayan cursado los dos tipos de estadística y algunas materias relacionadas con la misma.

Reconocen la necesidad de la estadística en su vida profesional y encuentran errores en las aplicaciones, lo cual despierta una necesidad adicional de reforzamiento del conocimiento. Para esto, sugieren cursos que atraigan su interés por el tema, además libros que permitan un mejor acercamiento a la materia.

2. En la componente razonamiento estadístico, se concluye que los jurados y/o tutores se ubican en los niveles de razonamiento pre-estructural y uniestructural del modelo de taxonomía "SOLO".

3. En la componente actitud se concluye que los tutores y/o jurados aunque tienen fobia hacia la estadística, y han aprendido en forma negativa y traumática, tienen una disposición favorable hacia el aprendizaje y actualización de los conocimientos.

En términos generales se concluye que la comunidad de investigadores formada está por 
tutores, jurados y/o asesores de trabajos de grado en dos universidades venezolanas, no poseen cultura estadística, lo cual explica la problemática planteada. Este resultado muestra la necesidad de insertar en la formación académica de los investigadores la estadística como disciplina $\mathrm{y}$ como una herramienta que garantizará la calidad de los productos científicos producidos en el entorno académico.

Es necesario implementar mecanismos de control de calidad hacia los trabajos que se presentan, $y$ diseñar estrategias de enseñanza y aprendizaje, que permitan cambiar en los investigadores las malas concepciones que tienen sobre esta disciplina, la cual es una herramienta metodológica primordial en el análisis de datos de cualquier investigación científica.

\section{Referencias}

Bracho, K. (2011). Cultura investigativa y producción científica en universidades privadas del municipio Maracaibo del Estado Zulia. Trabajo de investigación. [Resumen en línea]. Universidad Rafael Belloso Chacín. Revista Electrónica de humanidades, educación $y$ comunicación social. Disponible en: http:// publicaciones.urbe.edu/index.php/REDHECS/ article/view/952/2998. [Consulta: 2015, octubre]

Batanero, C. (2001). Didáctica de la estadística. España: Universidad de Granada.

Duarte. (2014). Comprensión y razonamiento de profesores de matemáticas de bachillerato sobre conceptos estadísticos básicos. Perfiles educativos. Vol.XXXVI, núm.146, 2014. IISUE-UNAM.

Estrada, M. (2012). Análisis de actitudes y conocimientos estadísticos elementales en la formación del profesorado. Tesis de doctorado en la didáctica de las matemáticas y las ciencias elementales. [Documento en línea]. Universidad Autónoma de Barcelona. España. Disponible en http://hdl.handle.net/10803/4697. Consulta: 2015, Julio.

Gal, I (2002). Adults'statistical literacy: Meanings, components, responsibilities. Internactional Satatistical Review, 70, 1-25.

Garfield, Del Mas y Chance (2003). Using student's informal notions of variability to develop an understanding of formal measures of variability. In M. lovett \& P. Shah (Eds), Thinking with data (pp.117-148). Mahwah, NJ: Lawrence Erlbaum Associates.

Leal, J. (2009). La autonomía del sujeto investigador y la metodología de investigación. 2da. Edición. Venezuela: Azul intenso. C.A. Valencia, Venezuela.

Pinto, J. (2010). Conocimiento didáctico del contenido sobre la representación de datos estadísticos. [Resumen en línea]. Tesis doctoral del departamento de didáctica de las matemáticas y de las ciencias experimentales. Universidad de Salamanca. España. Disponible en http:// hdl.handle.net/10366/76546. [Consulta: 2015, julio]

Rockeach, M. (1968). Las creencias, actitudes y valores. Una teoría de organización y el cambio. San francisco: Jossey-Bass.

Palella, S. (2004). Metodología de la investigación cuantitativa.

Velásquez, L. (2008) «Las redes de investigación virtuales: propuesta de fomento y desarrollo de la cultura investigativa en las instituciones de educación superior» [artículo en línea]. Revista de Universidad y Sociedad del Conocimiento (RUSC). Vol. 4, n. ${ }^{\circ}$ 2. UOC ISSN 1698-580X. Disponible en: http://www.uoc.edu/rusc/4/2/dt/ esp/velasquez.pdf. [Consulta: 2015, agosto]

Wise, S. (1985). El desarrollo y la validación de una escala para medir actitudes sobre la estadística. Mediciones de estadística y psicología. 